\title{
BMJ Open Evaluation of influenza surveillance systems in sub-Saharan Africa: a systematic review protocol
}

\author{
Evanson Zondani Sambala, ${ }^{1,2}$ Duduzile Edith Ndwandwe, ${ }^{1}$ Loveness M Imaan, ${ }^{2,3}$ \\ Charles S Wiysonge ${ }^{1,4,5}$
}

To cite: Sambala EZ, Ndwandwe DE, Imaan LM, et al. Evaluation of influenza surveillance systems in subSaharan Africa: a systematic review protocol. BMJ Open 2019;9:e023335. doi:10.1136/ bmjopen-2018-023335

- Prepublication history for this paper is available online. To view these files, please visit the journal online (http://dx.doi. org/10.1136/bmjopen-2018023335).

Received 2 April 2018 Revised 8 November 2018 Accepted 13 November 2018

Check for updates

(C) Author(s) (or their employer(s)) 2019. Re-use permitted under CC BY-NC. No commercial re-use. See rights and permissions. Published by BMJ.

For numbered affiliations see end of article.

Correspondence to

Dr Evanson Zondani Sambala; evanson.sambala@mrc.ac.za

\section{ABSTRACT}

Introduction Influenza infrastructure systems are crucial for maintaining surveillance operations, and for mitigating and responding to the disease. The role of surveillance is to isolate and identify as rapidly as possible any new influenza strains and collate this information for the preparedness for, and response to, an impending influenza activity in humans. However, sources of surveillance information, particularly in Africa, are meagre. This systematic review will critically evaluate the existing influenza surveillance systems in sub-Saharan Africa. Method and analysis We will build multiple electronic database search strategies for use in PubMed, Scopus, African Journal Online, Web of Science and Google scholar to identify as many studies as possible. The medical subject heading and keywords will include a wide range of synonyms, both in index terms and freetext words. Database search will be followed by hand searching of reference lists of all relevant studies. We will include eligible full-text studies published from 2002 in order to coincide with the establishment of the integrated disease surveillance and response system in Africa by WHO. We will examine the influenza surveillance performance systems using the US Centers for Disease Control and Prevention guidelines on evaluating public health surveillance systems. Our outcome measures will include surveillance system attributes such as timeliness, sensitivity, specificity, acceptability, representativeness, simplicity and usefulness. We will conduct a narrative synthesis of all studies.

Ethics and dissemination This study does not require ethics approval because it uses publicly available data. Our findings will be published in a peer review journal and disseminated to policy makers.

PROSPERO registration number CRD42018103042.

\section{INTRODUCTION}

The overarching goal of influenza surveillance systems is to isolate and identify as quickly as possible any new influenza strains and collate this information for control and mitigation of the impacts of influenza in humans. ${ }^{1}$ However, measuring influenza cases in real time is often a challenge because influenza viruses tend to undergo continuous antigenic variations, either bymutation

\section{Strengths and limitations of this study}

- Extraction of information from eligible studies using a standard evaluation checklist will efficiently summarise findings on the sensitivity, quality and usefulness of surveillance systems.

- A comprehensive and thorough search of published and unpublished literature in any language will be included to minimise the risk for publication bias.

- The search strategy excludes stochastically simulated or computer-simulated modelling techniques, thus placing limitation on surveillance methods that potentially may have greater sensitivity and specificity.

(antigenic drift) or genetic recombination (antigenic shift). ${ }^{2}{ }^{3}$ WHO recommends continuous monitoring of influenza viruses during each influenza season to identify the virus strains circulating in the human population and track the burden of influenza-related illnesses. ${ }^{4}$ Surveillance further provides information on the candidate viruses for vaccine production and monitoring of antiviral sensitivity. ${ }^{5}$

Surveillance of influenza is an important public health task. However, sources of surveillance information, particularly in sub-Saharan Africa, are insufficient. ${ }^{16}$ There are undoubtedly general difficulties in surveillance systems in Africa, in the robustness of the data needed to send early signals for health service response and in the financial resources and leadership required for efficient and timely decision making. ${ }^{7}$ Influenza surveillance is persistently characterised by low completeness and timeliness in reporting due to inadequate laboratories, communication structures and poor public health human resources. ${ }^{7}$ A related challenge, despite the use of standard case definitions of influenza-like illness (ILI) and severe acute respiratory infections (SARIs), is sampling issues within surveillance systems, which are often 
biased towards collecting more specimens from patients suspected to have influenza on clinical grounds. ${ }^{8}$ In some cases, calculation of death associated to ILI and SARI is difficult to ascertain especially where such deaths are not attributable to influenza illness as the primary cause.

Epidemiological methods using inferential statistics can explain epidemiological events, and are important for any public health decisions that inform influenza prevention and control strategies. Indirect indices such as excess mortality, attack rates and clinical symptoms are signature measures of the impact of influenza. A measure of excess deaths related to influenza remain an issue, the mathematical models used to calculate it are no means a direct one. Epidemiologists in seasonal climates determine excess deaths attributable to influenza, including influenza burden on hospitalisation (during winter months), by use of Serfling-like cyclical regression models and Auto Regressive Integrated Moving Average (ARIMA) models. ${ }^{19}$ However, these models are not applicable in tropical climates where seasonality is all year round whereas these methods require cyclic seasonal patterns of viral activity interrupted by influenza-free periods. Due to these limitations, other methods are deployed in order to quantify influenza surveillance data, such as use of the influenza pyramid of severity, the spread of disease and risk factor determination, which often do not require emphasis on viral culture and laboratory isolation.

Influenza infrastructure systems are crucial for maintaining operations of surveillance, and for mitigating and responding to the disease. WHO defines surveillance systems as a "continuous, systematic collection, analysis and interpretation of health-related data needed for the planning, implementation, and evaluation of public health practice'. ${ }^{10}$ However, there is little agreement as to which sources of surveillance data are most appropriate for early and sensitive warning of influenza outbreaks and management.

This systematic review critically evaluates the existing surveillance systems used to collect and analyse surveillance data for detecting when and where influenza activity will occur. Our findings will inform public health policy makers about the role of strengthening surveillance systems.

\section{OBJECTIVE}

To evaluate and describe influenza surveillance systems designed to detect the occurrence, circulation and impacts of influenza-related illnesses.

\section{METHODS}

\section{Eligibility criteria}

Types of studies to be included

Observational studies on influenza surveillance systems or methods that describe or evaluate the collection, analysis and reporting of influenza surveillance. We will include the following categories of surveillance systems:
- Virological surveillance, for example, public health and clinical laboratories that test specimens to understand when and where influenza viruses are circulating.

- Outpatient illness surveillance, for example, patient visits to healthcare providers for ILI/contact tracing.

- Mortality surveillance, for example, influenza-associated deaths.

- Hospitalisation surveillance, for example, laboratory-confirmed influenza-associated hospitalisations.

- Geographical spread of influenza, for example, no activity, sporadic, local, regional or widespread.

All studies that report mathematical models or use hypothetical surveillance data will be excluded.

\section{Types of participants}

This review will draw on all human participants. Although young children, the elderly and persons with underlying medical conditions are at higher risk of severe influenza, the disease can affect any individual. For example, the 2009 pandemic H1N1 virus remained antigenically unchanged in May 2012, still affecting young adults (as in the 2010/2011 season) as seasonal influenza. ${ }^{11}$ Surveillance systems are designed to detect the disease in all individuals for its prevention and control. All studies reporting on animal surveillance systems will be excluded.

\section{Types of exposure}

Identifying and quantifying influenza cases is generally more challenging compared with other infectious diseases, which have clear-cut clinical signs. Influenza illnesses, especially mild ones, may go unreported and sometimes may warrant no medical attention. ${ }^{1}$ Influenza virus infections can lead to fatal complications and death associated with pre-existing medical conditions such as cardiovascular diseases. ${ }^{12}$ In cases of death associated with pre-existing medical conditions, influenza would not be typically identified as the primary cause of death. We will adopt the WHO case definitions for ILI and SARI. ILI is defined as 'an acute respiratory illness with measured temperature of $\geq 38^{\circ} \mathrm{C}$ and cough, with onset within 10 days ${ }^{13}$ while SARI is defined as 'an acute respiratory illness with a history of fever or measured fever of $\geq 38^{\circ} \mathrm{C}$ and cough, with onset within the past 10 days, requiring hospitalisation'. ${ }^{13}$

\section{Types of the outcomes measures}

The primary outcomes include the following influenza surveillance system attributes:

1. Simplicity, that is, ease of operation.

2. Flexibility, that is, adapting to changing information such as case definitions.

3. Data quality, that is, completeness and validity.

4. Acceptability, that is, willingness of staff to use the system.

5. Sensitivity, that is, timely detection of influenza.

6 . Positive predictive value, that is, proportion of true cases. 
7. Representativeness, that is, distribution in the population by place and person

8. Timeliness, that is, speed between steps in a public health surveillance system.

9. Stability, that is, the ability to collect, manage and provide data properly without failure.

The secondary outcome measures will include the following descriptions of the surveillance system tasks:

1. Public health importance.

2. System under evaluation.

3. Resources to operate the surveillance system.

4. Usefulness of the surveillance system.

5. Design.

6. Conclusion and recommendations of the system.

7. Utilisation of the findings and how these are shared as lessons learnt with stakeholders.

\section{Search strategy}

We will build multiple electronic database search strategies for use in PubMed, Scopus, African Journal Online, Web of Science and Google scholar to identify as many titles as possible in any language. The medical subject heading terms ILI and SARI, surveillance, virological, outpatient illness, mortality, morbidity, hospitalisation, laboratory confirmed, influenza, sentinel, seasonal, pandemic, infrastructures and Africa will be used to combine searches systematically. The search terms will include a wide range of synonyms, both in index terms and free-text words. The exact terms for the search in PubMed is presented in box 1. Hand searching of reference lists of all relevant studies and reports will be completed to identify additional studies that might be eligible for the review. All the identified titles and abstracts will be screened for inclusion. If relevant, a full-text article will be obtained and read carefully. The titles searched by hand from the reference lists will be retained into the database search for a full text. We will search for studies published from 01 January 2002 to coincide with the establishment of the integrated disease surveillance and response system by WHO.

\section{Searching other resources}

We will identify country-eligible reports or preliminary analyses from respective national influenza centres and FluNet where surveillance data are shared. We will search the websites of relevant government agencies, the WHO's Global Influenza Surveillance and Response System, Global Influenza Surveillance Network and Global Influenza Programme. We will also search conference reports and abstracts from the African Network for Influenza Surveillance and Epidemiology and the Africa Influenza Alliance Meeting.

\section{Selection of studies}

Two authors (EZS and DEN) will screen titles and abstracts for eligibility independently of each other based on the inclusion criteria. We will import the identified titles and abstracts into the reference database using EndNote

\section{Box 1 Search strategy}

((()(((/'africa'[MeSH Terms] OR 'africa'[All Fields]) OR ('africa south of the sahara'[MeSH Terms] OR ('africa'[All Fields] AND 'south'[All Fields] AND 'sahara'[All Fields]) OR 'africa south of the sahara'[All Fields] OR ('sub'[All Fields] AND 'saharan'[All Fields] AND 'africa'[All Fields]) OR 'sub saharan africa'[All Fields])) OR ('africa, western'[MeSH Terms] OR ('africa'[All Fields] AND 'western'[All Fields]) OR 'western africa'[All Fields] OR ('west'[All Fields] AND 'africa'[All Fields]) OR 'west africa'[All Fields])) OR ('africa, northern' [MeSH Terms] OR ('africa'[All Fields] AND 'northern'[All Fields]) OR 'northern africa'[All Fields] OR ('north'[All Fields] AND 'africa'[All Fields]) OR 'north africa'[All Fields])) OR ('africa, southern'[MeSH Terms] OR ('africa'[All Fields] AND 'southern'[All Fields]) OR 'southern africa'[All Fields] OR ('southern'[All Fields] AND 'africa'[All Fields]))) OR ('africa, central'[MeSH Terms] OR ('africa'[All Fields] AND 'central'[All Fields]) OR 'central africa'[All Fields] OR ('central'[All Fields] AND 'africa'[All Fields]))) OR ('africa, eastern'[MeSH Terms] OR ('africa'[All Fields] AND 'eastern'[All Fields]) OR 'eastern africa'[All Fields] OR ('east'[All Fields] AND 'africa'[All Fields]) OR 'east africa'[All Fields]))

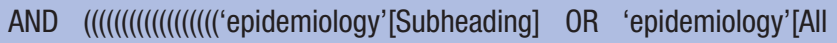
Fields] OR 'surveillance'[All Fields] OR 'epidemiology'[MeSH Terms] OR 'surveillance'[All Fields]) OR (('epidemiology'[Subheading] OR 'epidemiology'[All Fields] OR 'surveillance'[All Fields] OR 'epidemiology'[MeSH Terms] OR 'surveillance'[All Fields]) AND systems[All Fields])) OR (('epidemiology'[Subheading] OR 'epidemiology'[All Fields] OR 'surveillance'[All Fields] OR 'epidemiology'[MeSH Terms] OR 'surveillance'[All Fields]) AND types[All Fields])) OR sentinel[All Fields]) OR ('virology'[MeSH Terms] OR 'virology'[All Fields] OR 'virologic'[All Fields])) OR infrastructure[All Fields]) OR ('mortality'[Subheading] OR 'mortality'[All Fields] OR 'mortality'[MeSH Terms])) OR ('epidemiology'[Subheading] OR 'epidemiology'[All Fields] OR 'morbidity'[All Fields] OR 'morbidity'[MeSH Terms])) OR (pyramid[All Fields] AND severity[All Fields])) OR (('risk factors' [MeSH Terms] OR ('risk'[All Fields] AND 'factors'[All Fields]) OR 'risk factors'[All Fields] OR ('risk'[All Fields] AND 'factor'[All Fields]) OR 'risk factor'[All Fields]) AND ('analysis'[Subheading] OR 'analysis'[All Fields] OR 'determination'[All Fields]))) OR (spread[All Fields] AND ('disease'[MeSH Terms] OR 'disease'[All Fields]))) OR (('virology'[MeSH Terms] OR 'virology'[All Fields] OR 'viral'[All Fields]) AND ('ethnology'[Subheading] OR 'ethnology'[All Fields] OR 'culture'[All Fields] OR 'culture'[MeSH Terms]))) OR (('laboratories'[MeSH Terms] OR 'laboratories'[All Fields] OR 'laboratory'[All Fields]) AND ('isolation and purification'[Subheading] OR ('isolation'[All Fields] AND 'purification'[All Fields]) $\mathrm{OR}$ 'isolation and purification'[All Fields] OR 'isolation'[All Fields]))) OR (('death'[MeSH Terms] OR 'death'[All Fields]) AND attributable[All Fields])) OR (('laboratories' [MeSH Terms] OR 'laboratories'[All Fields] OR 'laboratory'[All Fields]) AND confirmed[All Fields])) OR ('hospitalisation'[All Fields] OR 'hospitalisation'[MeSH Terms] OR 'hospitalisation'[All Fields])) OR admission[All Fields]) OR ('outpatients'[MeSH Terms] OR 'outpatients'[All Fields]))) AND ((((((seasonal[All Fields] OR ('pandemics'[MeSH Terms] OR 'pandemics'[All Fields] OR 'pandemic'[All Fields])) OR ('influenza, human'[MeSH Terms] OR ('influenza'[All Fields] AND 'human'[All Fields]) OR 'human influenza'[All Fields] OR 'influenza'[All Fields])) OR (('influenza, human'[MeSH Terms] OR ('influenza'[All Fields] AND 'human'[All Fields]) OR 'human influenza'[All Fields] OR 'influenza'[All Fields]) AND like[All Fields] AND illness[All Fields])) OR (severe[All Fields] AND acute[All Fields] AND ('respiratory tract infections'[MeSH Terms] OR ('respiratory'[All Fields] AND 'tract'[All Fields] AND 'infections'[All Fields]) OR 'respiratory tract infections'[All Fields] OR ('respiratory'[All Fields] AND 'infections'[All Fields]) OR 'respiratory infections'[All Fields]))) OR SARI[All Fields]) OR ILI[All Fields])) AND ((((serfling[All Fields] AND like[All Fields] AND cyclical[All Fields] AND

Continued 


\section{Box 1 Continued}

('regression (psychology)'[MeSH Terms] OR ('regression'[All Fields] AND '(psychology)'[All Fields]) OR 'regression (psychology)'[All Fields] OR 'regression'[All Fields]) AND ('Model Driven Eng Lang Syst'[Journal] OR 'models'[All Fields])) OR (('moclobemide'[MeSH Terms] OR 'moclobemide'[All Fields] OR 'arima'[All Fields]) AND ('Model Driven Eng Lang Syst'[Journal] OR 'models'[All Fields]))) OR ('transmission'[Subheading] OR 'transmission'[All Fields])) OR (cyclinic[All Fields] AND seasonal[All Fields] AND patterns[All Fields]))

MeSH, medical subject heading.

V.X7. The full text of potentially eligible studies will be screened for duplications by two independent authors (EZS and DEN). All the duplicate articles and reports will be removed. Where disagreement arises on the study eligibility due to lack of information on the study manuscript or report, we will contact the authors of the article for additional information or clarification. All discrepancies and disagreements will be resolved by consensus and, if necessary, by arbitration of the third author (CSW). We will translate words, phrases and web pages into English from other languages using the Google translation software.

\section{Data extraction and management}

We will examine studies and reports that describe surveillance systems for collecting, analysing or presenting surveillance data for influenza-related illnesses or syndromes. Two independent authors (EZS and DEN) will extract information from eligible studies using a standard data extraction form shown in table 1 . In addition, we will extract details from studies and reports such as the year of study, settings, study design, methods, participants, source of funding and risk of bias. Two authors will compare the extracted data and resolve discrepancies or any disagreements by discussion to reach consensus. If the two authors are unable to resolve disagreements a third author (CSW) will be consulted. Where there are missing data in the studies and reports, we will contact the study author(s) for additional information.

\section{Assessment of the risk of bias in the included studies}

Two independent authors (EZS and DEN) will assess the risk of bias in observation studies using the Risk of Bias In Non- Randomised Studies -of Interventions (ROBINS-I) tool. ${ }^{14}$ We will evaluate the following domains for risk of bias: confounding, selection of participants, classification of interventions, deviations from intended interventions, missing data, measurement of outcomes and selection of

\begin{tabular}{|c|c|c|c|c|c|c|c|}
\hline Category & Criteria & 1 & 2 & 3 & 4 & 5 & NA \\
\hline \multirow[t]{2}{*}{ Stakeholders } & $\begin{array}{l}\text { Describe persons or organisations who will use the } \\
\text { data gathered by the surveillance system. } \\
\text { Cape }\end{array}$ & & & C & $\mathrm{Ca}$ & & Cap \\
\hline & Describe resources required to operate the system. & & & & & & \\
\hline \multirow[t]{3}{*}{ Evaluation design } & Describe the specific purpose of the evaluation. & & & & & & \\
\hline & Propose uses of the evaluation results. & & & & & & \\
\hline & $\begin{array}{l}\text { Include the specific questions that the evaluation } \\
\text { answered. }\end{array}$ & & & & & & \\
\hline \multirow[t]{11}{*}{ Credible evidence } & $\begin{array}{l}\text { Describe usefulness of the information generated by } \\
\text { analysing data. }\end{array}$ & & & & & & \\
\hline & Assess surveillance system attributes in terms of: & & & & & & \\
\hline & Simplicity & & & & & & \\
\hline & Flexibility & & & & & & \\
\hline & Data quality & & & & & & \\
\hline & Acceptability & & & & & & \\
\hline & Sensitivity & & & & & & \\
\hline & Positive predictive value & & & & & & \\
\hline & Representativeness & & & & & & \\
\hline & Timeliness & & & & & & \\
\hline & Stability & & & & & & \\
\hline $\begin{array}{l}\text { Conclusions and } \\
\text { recommendations }\end{array}$ & $\begin{array}{l}\text { Present conclusions based on evaluation results. } \\
\text { Propose appropriate and applicable recommendations } \\
\text { to improve the system or discontinue its operation. }\end{array}$ & & & & & & \\
\hline Lessons learnt & $\begin{array}{l}\text { Describe how lessons learnt will be communicated to } \\
\text { those who need to know. }\end{array}$ & & & & & & \\
\hline
\end{tabular}




\begin{tabular}{ll}
\hline Table 2 Scoring sheet \\
\hline Points & Description \\
\hline $5=$ excellent & $\begin{array}{l}\text { The element is present, consistent } \\
\text { with the standard described in } \\
\text { the instructions and provided in the } \\
\text { classroom, and is of a remarkable/ } \\
\text { outstanding quality. }\end{array}$ \\
$\begin{array}{l}\text { The element is present and consistent } \\
\text { with the standard described in the } \\
\text { instructions and provided in the } \\
\text { classroom. }\end{array}$ \\
$\begin{array}{l}\text { The element is present and may be } \\
\text { used even though it may not completely } \\
\text { follow the standard described in } \\
\text { the instructions and provided in the } \\
\text { classroom. }\end{array}$ \\
$\begin{array}{l}\text { The element is present but flawed or of } \\
\text { poor quality. }\end{array}$ \\
$\begin{array}{l}\text { The element is absent from the report. } \\
\text { T=absent }\end{array}$
\end{tabular}

the reported results. We will develop a risk of bias table to summarise our assessments. In the context of surveillance systems, biases arise in case definitions (ie, SARI/ILI) and the type of diagnostic equipment (Reverse Transcription Polymerase Chain Reaction (RT-PCR) versus immunofluorescence assays) used to test influenza as these variations can lead to selection or misclassification bias in the surveillance techniques. Any disagreements or discrepancies concerning the risk of bias will be resolved by discussion and if any disagreement cannot be resolved, an involvement of a third reviewer (CSW) will be recommended.

\section{Data synthesis}

We will score each surveillance task including its element by assigning values from 5 (excellent) to 1 (absent). Table 2 shows the point scoring criteria for evaluating the studies and reports. We will describe the surveillance performance system using US Centers for Disease Control and Prevention guidelines on evaluating public health surveillance systems. ${ }^{1516}$ First developed in 1988 and updated in 2001, the guidelines ensure that the influenza surveillance system operates efficiently and effectively in meeting its intended purpose and objectives. In order to gauge the strength of these surveillance systems we will sum up the scores for each surveillance system using averages and percentages. In addition, we will also generate and organise data using the predefined themes captured in the data collection form.

\section{Reporting of this review}

We designed and wrote this study protocol following the Preferred Reporting Items for Systematic Review and Meta-Analysis Protocols (PRISMA-P) guidelines. ${ }^{17}$ We considered all the 17 items in PRISMA-P to facilitate transparent and complete reporting of systematic reviews and metaanalyses. We will report the results of this review according to guidance contained in the PRISMA statement. ${ }^{17}$ Any amendments to the protocol will subsequently be updated in the International prospective register of systematic reviews (PROSPERO).

\section{Time line for the systematic review}

We plan to conduct the searches and select eligible studies between November and December 2018. We hope to complete the data analysis, write up and submit the first draft of the manuscript by December 2018.

\section{Patient and public involvement}

Patients were not involved in the design of this study. The study does not require the recruitment of patients and/or for the public to be involved as this systematic review only assesses available influenza surveillance systems.

\section{DISCUSSION}

This protocol for systematic review will evaluate influenza surveillance systems using available literature. We will explore the existing types of surveillance tools used to gather data and evaluate study techniques used in surveillance reporting and monitoring of influenza. We hope to identify gaps in our findings and recommend directions for future evaluation of systems. In addition, our findings will enhance the utility to respond rapidly to the early warning signs of new influenza viruses and subsequently strengthen the overall surveillance systems by informing public health policy. Influenza surveillance systems, particularly those designed for seasonal influenza outbreaks, are important. They can serve as a predictive indicator for new events such as an influenza pandemic, and systematically aid pandemic planners in planning for additional capacities and resources needed to deal with a more severe outbreak. ${ }^{18}$

There are potential limitations to this study. First, we anticipate that reported surveillance systems at the country level will lack geographical representativeness because sentinel data aggregated at the national influenza centres are often unevenly distributed. Subsequently, this may introduce bias in our review findings. Second, our search strategy excludes stochastically simulated and computer-simulated modelling techniques yet these methods have high sensitivity and specificity. This substantially limits our understanding of the types of surveillance systems that may exist. Third, we use a data extraction tool specifically developed for the surveillance systems in USA to describe and assess the strength of surveillance systems in Africa. With such a limitation, our findings may not be a true reflection of the established surveillance systems in Africa.

\section{Author affiliations}

${ }^{1}$ Cochrane South Africa, South African Medical Research Council, Tygerberg, Cape Town, Western Cape, South Africa

${ }^{2}$ Malawi Public Health Forum, Lilongwe, Malawi

${ }^{3}$ Social Work Department, Catholic University of Malawi, Blantyre, Malawi

${ }^{4}$ Centre for Evidence-based Health Care, Division of Epidemiology and Biostatistics, Department of Global Health, Faculty of Medicine and Health Sciences, Stellenbosch University, Cape Town, South Africa 
${ }^{5}$ Division of Epidemiology and Biostatistics, School of Public Health and Family Medicine, University of Cape Town, Cape Town, South Africa

Contributors EZS, DEN, LMI and CSW contributed to the conceptualisation of the review. EZS wrote the manuscript draft. EZS and LMI developed the search strategy. All authors revised and edited the manuscript draft and search strategy. All authors approved the manuscript.

Funding The authors have not declared a specific grant for this research from any funding agency in the public, commercial or not-for-profit sectors.

Competing interests None declared.

Patient consent for publication Not required.

Provenance and peer review Not commissioned; externally peer reviewed.

Open access This is an open access article distributed in accordance with the Creative Commons Attribution Non Commercial (CC BY-NC 4.0) license, which permits others to distribute, remix, adapt, build upon this work non-commercially, and license their derivative works on different terms, provided the original work is properly cited, appropriate credit is given, any changes made indicated, and the use is non-commercial. See: http://creativecommons.org/licenses/by-nc/4.0/.

\section{REFERENCES}

1. Sambala EZ. Ethics of planning for, and responding to, pandemic influenza in Sub Saharan Africa: qualitative study: University of Nottingham, 2014.

2. Sleman S. How Influenza a causes "Epidemics and Pandemics" among the population: novel targets for anti-influenza molecules. $J$ of Epi Inf Dis 2017;1.

3. Cliff AD, Haggett P, Ord JK. Spatial aspects of influenza epidemics: Routledge, 1986.

4. World Health Organization. WHO interim global epidemiological surveillance standards for influenza. Geneva: World Health Organization, 2012:1-61.
5. Tosh PK, Jacobson RM, Poland GA. Influenza vaccines: from surveillance through production to protection. Mayo Clin Proc 2010;85:257-73.

6. Schoub BD. Surveillance and management of influenza on the African continent. Expert Rev Respir Med 2010;4:167-9.

7. Katz MA, Schoub BD, Heraud JM, et al. Influenza in Africa: uncovering the epidemiology of a long-overlooked disease. J Infect Dis 2012;206:S1-4.

8. Radin JM, Katz MA, Tempia S, et al. Influenza surveillance in 15 countries in Africa, 2006-2010. J Infect Dis 2012;206:S14-21.

9. Simonsen L, Viboud C, Taylor RJ, et al. The epidemiology of influenza and its control. In Influenza vaccines for the future: Springer, 2011:27-54.

10. World Health Organization. Public health surveillance. 2018. http:// www.who.int/immunization/monitoring_surveillance/burden/vpd/en/ (Cited 5 Jul 2018).

11. Mytton OT, Rutter PD, Donaldson LJ. Influenza $A(H 1 N 1) p d m 09$ in England, 2009 to 2011: a greater burden of severe illness in the year after the pandemic than in the pandemic year. Euro Surveill 2012;17:20139.

12. Finelli L, Chaves SS. Influenza and acute myocardial infarction. $J$ Infect Dis 2011;203:1701-4.

13. Fitzner J, Qasmieh S, Mounts AW, et al. Revision of clinical case definitions: influenza-like illness and severe acute respiratory infection. Bull World Health Organ 2018;96:122-8.

14. Sterne JA, Hernán MA, Reeves BC, et al. ROBINS-I: a tool for assessing risk of bias in non-randomised studies of interventions. BMJ 2016;355:i4919.

15. Klaucke DN, Buehler JW, Thacker SB, et al. Guidelines for evaluating surveillance systems. MMWR Suppl 1988;37:1-18.

16. German RR, Lee L, Horan J, et al. Updated guidelines for evaluating public health surveillance systems. MMWR Recomm Rep 2001;50:1-35.

17. Moher D, Shamseer L, Clarke M, et al. Preferred reporting items for systematic review and meta-analysis protocols (PRISMA-P) 2015 statement. Syst Rev 2015;4:1.

18. World Health Organization. Pandemic Influenza Risk Management WHO Interim Guidance: World Health Organization, 2013. (Cited 5Jul 2018). 